\title{
Polarization effects in early SUSY searches at the CERN LHC
}

\author{
Kai Wang ${ }^{\mathrm{a}}$, Liucheng Wang, Tao Xu, Liangliang Zhang \\ Department of Physics, Zhejiang Institute of Modern Physics, Zhejiang University, Hangzhou 310027, Zhejiang, China
}

Received: 26 June 2014 / Accepted: 10 June 2015 / Published online: 26 June 2015

(C) The Author(s) 2015. This article is published with open access at Springerlink.com

\begin{abstract}
An on-shell effective theory (OSET) approach has been widely used in searches of various supersymmetric signals, in particular, gluino/squark pairs with long cascade decay chains in which complete matrix element calculations may encounter high dimensional integrations. On the other hand, leptons from polarized chargino decays may show a significant boost effect in some scenarios and simulation without polarization information may underestimate or overestimate the lepton $p_{\mathrm{T}}$ cut efficiencies in the first place. We study the polarization effects in leptonic decaying charginos from squarks or gluinos. Taking the polarization effects into account, we find it still justifiable to take only the OSET approach for a large parameter region, for instance, the first two generation squarks due to indistinguishable final states as well as a flat angular distribution in the motion of the lepton. On the other hand, we use the leptonic stop to illustrate the feature and find that the lepton $p_{\mathrm{T}}$ cut efficiencies in cross section measurements can have maximally $25 \%$ reduction or maximally $17 \%$ enhancement in comparison with the kinematics-only approach. The signal rates after the cuts simulated by OSET are then underestimated/overestimated and the real bound on the squark/gluino should be more stringent or loose for a specific choice of the chargino and one can take the simulated efficiencies as a fast-simulation factor to multiply to the OSET simulated results.
\end{abstract}

\section{Introduction}

With two years' running, the Large Hadron Collider (LHC) at CERN has accumulated data of $30 \mathrm{fb}^{-1}$ integrated luminosity. In the early stage, most of the discoveries were kinematics dominated. On-shell effective theories (OSET) which characterize hadron collider data in terms of masses, production cross sections, and decay modes of candidate new particles play an important role in new physics searches [1]. In princi-

\footnotetext{
a e-mail: wangkai1@zju.edu.cn
}

ple, if the precision predictions of the production cross section and decay mode for a given mass of a candidate new particle exist, one can also obtain information on its spin. However, direct confirmation of the spin state measurement only comes from a measurement of the angular correlation. With only about $28 \mathrm{fb}^{-1}$, a Higgs boson has been discovered with over $7 \sigma$ significance via measurement of the invariant masses for four-lepton final states. Both the ATLAS and the CMS collaborations have found a resonance of four leptons with an invariant mass of $125 \mathrm{GeV}$ and the reconstructed diphoton invariant mass also peaks at the same place. The diphoton final state has excluded the boson to be a spin- 1 state based on an argument by the Landau-Yang theorem [2,3]. The further analysis of the spin/parity measurement of the boson based on data on the angular correlation in four-lepton channel is very compatible with the scalar boson expectations of $0^{+}$. The data disfavors the $2^{+}$hypothesis with a confidence level value of $0.6 \%$ [4,5]. Whether the boson is the standard model (SM) Higgs then requires additional measurements of its Yukawa couplings to the SM fermions.

On the other hand, the transverse momentum distribution $d \sigma / d p_{\mathrm{T}}$ is not only a result of the mass spectrum but also dependent on polarization of spin- 1 or spin- $1 / 2$ particles, while the measurement of the cross section significantly depends on the simulated cut efficiency. Consequently, the polarization effect may in principle enter for the very early stage measurement of the cross section.

A full matrix element simulation automatically contains all spin and polarization information. However, in many cases, searches may involve multi-body final states which correspond to a multi-body phase-space integration. An $n$ body phase space is a $3 n-4$ dimensional integration with the additional two dimensions over the two initial parton distributions, so a $3 n-2$ dimensional integration is then required. High dimensional integration is technically extremely challenging. Therefore, simulation based on kinematic decay is sometimes inevitable. For instance, gluino cascade decay into the lepton final state 
$\tilde{g} \rightarrow j j \chi_{1}^{ \pm} \rightarrow j j \ell^{ \pm} \nu_{\ell} \tilde{\chi}_{1}^{0}$

involves five-body final states in one chain. In $R$-parity conserving supersymmetric standard models, gluino pair production with di-lepton final states then corresponds to the ten-body case, and the total integration is a 28 dimensional integration $[6,7]$. The di-lepton plus jets arising from squark pair or gluino-squark production then corresponds to eightor nine-body final states and 22 or 25 dimensional integration. The simulations of these final states are typically done by only kinematics in Pythia. In this paper, we study how the polarization effect changes the measurement of the production cross section in particular. We compare the lepton $p_{\mathrm{T}}$ cut efficiency between a study involving polarization and a study with pure kinematic decays in different scenarios.

In the next section, we first discuss the $p_{\mathrm{T}}$ distribution as a result of polarization. Then we discuss the chargino polarization in squark/gluino decay and its leptonic decay distribution in different scenarios, for instance, light sleptons, off-shell $W$ or on-shell $W$. We then give the numeric results of the comparison before we conclude.

\section{$2 p_{\mathrm{T}}$ Distribution as polarization effects}

We use two examples to illustrate the polarization effects on the $p_{\mathrm{T}}$ distributions. In a one-prong decay of $\tau$ lepton $\tau^{-} \rightarrow \pi^{-} \nu_{\tau}$, the nearly massless $\nu_{\tau}$ is of left-handed helicity and the pion $\pi^{-}$is a pseudo-scalar state. In the left-handed $\tau_{\mathrm{L}}^{-}$rest frame, the neutrino $\nu_{\tau}$ is moving in the direction of the $\tau_{\mathrm{L}}^{-}$boost direction and the pion is boosted in the opposite direction of the $\tau_{\mathrm{L}}^{-}$direction of the movement. For the righthanded $\tau_{\mathrm{R}}^{-}$, the pion $\pi^{-}$is boosted in the $\tau_{\mathrm{R}}^{-}$direction of the movement. Therefore, a left-handed or right-handed $\tau$ can be clearly distinguished via the $p_{\mathrm{T}}$ measurement of the pions. This feature can be applied to a search of the new charged Higgs state $H^{ \pm}$, since the $\tau^{ \pm}$from a $H^{ \pm}$decay and the leading irreducible background of $W^{ \pm}$decay are in different helicity states [8]. A simple $p_{\mathrm{T}}$ cut in the charged pion of the $\tau$-tag can significantly reduce the SM background from $W^{ \pm}$ decay.

The second example is the $W$-polarization measurements with a $p_{\mathrm{T}}$ cut. We plot the $p_{\mathrm{T}}$ cut effects over the measurement of $W$-polarization in the top-quark decay $t \rightarrow$ $b W^{+}[9,10]$. In the Higgs mechanism, the Goldstone degree of freedom becomes the longitudinal polarization of the $W$ boson, $\epsilon^{0}$. Since the top quark is the heaviest particle which acquires mass through electroweak symmetry breaking (EWSB), the top quark couples to the Goldstone boson strongly with $m_{t} / m_{W}$ enhancement in $\epsilon_{\mu}^{0 *} \bar{u}_{b} P_{\mathrm{L}} \gamma^{\mu} u_{t} \propto$ $m_{t} / m_{W}$. One uses the helicity fractions $F_{0}, F_{\mathrm{L}}$, and $F_{\mathrm{R}}$ to denote the fractions of longitudinal, left-handed, and righthanded $W$ polarizations, respectively. Due to the $m_{t} / m_{W}$

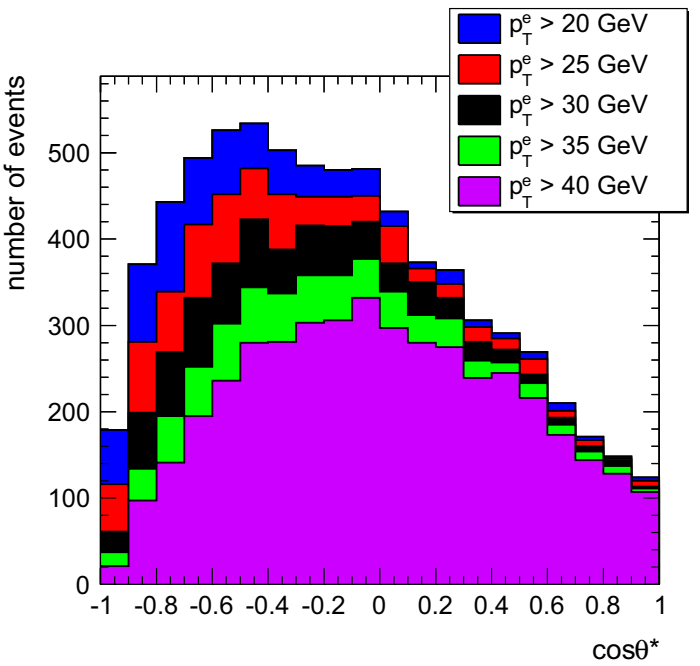

Fig. $1 d \Gamma / d \cos \theta$ distribution in SM semi-leptonic $t \bar{t}$ with different $p_{\mathrm{T}}$ selection cuts as labeled in plot

enhancement, the SM predictions are $F_{0} 0.688 \pm 0.004$, $F_{\mathrm{L}}=0.310 \pm 0.004$, and $F_{\mathrm{R}}=0.0017 \pm 0.0001$ [11]. Given the full reconstruction of the semi-leptonic $t \bar{t}$ system, polarizations can be measured by measuring the angle between the lepton and $W$ in the $W$ rest frame. In the left-handed polarized $W^{+} W^{+} \rightarrow\left(e^{+}\right)_{\mathrm{R}}\left(v_{e}\right)_{\mathrm{L}}$ case, the lepton is boosted in the opposite direction of the movement of $W^{+}$with

$$
\left|\mathcal{M}\left(W_{\mathrm{L}}^{+} \rightarrow e^{+} v_{e}\right)\right|^{2} \propto(1-\cos \theta)^{2}
$$

where $\theta$ is defined as the angle between the momentum direction of the lepton from $W$ and the reversed momentum direction of the bottom quark from top-quark decay, both boosted into the $W$-boson rest frame. In this case, the measured lepton momentum in the lab frame are softer than the ones in the $W$ rest frame and softer leptons may not pass the lepton $p_{\mathrm{T}}$ cut. Therefore, the portion of the $F_{\mathrm{L}}$ measurement clearly depends on the lepton $p_{\mathrm{T}}$ cut.

Figure 1 shows the $d \Gamma / d \cos \theta$ distribution in SM semileptonic $t \bar{t}$ with different $p_{\mathrm{T}}$ selection cuts as labeled in plot from $p_{\mathrm{T}}: 20--40 \mathrm{GeV}$.

\section{Leptonic decay of strongly interacting SUSY particles}

An on-shell effective theory approach plays a particularly important role in simulating multi-jet final states with the lepton arising from gluino or squark cascade decays due to the difficulty in multi-dimensional integration. Due to the small leptonic decay branching fraction, final states with multi-jet plus large $\not_{\mathrm{T}}$ with one or two leptons are the leading channels besides the pure hadronic channels. We therefore focus on such cascade decays with a mono-lepton, and the typical processes contain the lightest chargino $\tilde{\chi}_{1}^{ \pm} \rightarrow \ell^{ \pm} v \tilde{\chi}_{1}^{0}$ of a polarized $\tilde{\chi}_{1}^{ \pm}$. 


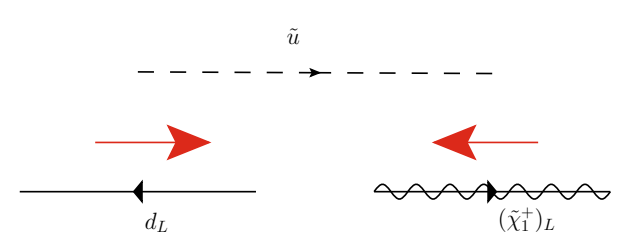

Fig. 2 Spin correlation in squark decaying into charging, left-handed polarized $\tilde{\chi}_{1}^{+}$

Chargino states are a mixture of both wino and Higgsino, which is determined by $M_{2}$ and $\mu$ in the mixing matrix. We first discuss the wino chargino for general first two generation squark decay. Suppressed by the light quark Yukawa, the decay BR into a Higgsino of the squark is only significant for third generations and we discuss this as a special case below.

The wino is a super-partner of the $S U(2)_{\mathrm{L}}$ weak gauge boson $W$, and the squark-quark-wino vertex

$\mathcal{L}=\left[-g V_{11} \tilde{u}_{\mathrm{L}}\right] \bar{d} P_{\mathrm{R}} \tilde{\chi}_{1}^{+c}$

Corresponds to the weak interaction, where only lefthanded quark or right-handed anti-quark participates the interaction. ${ }^{1}$ As shown in Fig. 2, the left up-type squark $\tilde{u}_{\mathrm{L}}$ decays into a left-handed $d$-quark, which results in $\tilde{\chi}_{1}^{+}$to be only left-handed. ${ }^{2}$

When the chargino is a wino, for all squarks and antisquarks, one can write down similar relations,

$$
\begin{array}{ll}
\tilde{u}_{\mathrm{L}} \rightarrow d_{\mathrm{L}}\left(\tilde{\chi}_{1}^{+}\right)_{\mathrm{L}}, & \tilde{u}_{\mathrm{L}}^{*} \rightarrow(\bar{d})_{\mathrm{R}}\left(\tilde{\chi}_{1}^{-}\right)_{\mathrm{R}} \\
\tilde{d}_{\mathrm{L}} \rightarrow u_{\mathrm{L}}\left(\tilde{\chi}_{1}^{-}\right)_{\mathrm{L}}, & \tilde{d}_{\mathrm{L}}^{*} \rightarrow(\bar{u})_{\mathrm{R}}\left(\tilde{\chi}_{1}^{+}\right)_{\mathrm{R}} .
\end{array}
$$

On the other hand, for the first two generations, scalar quarks decay into light quark states which hadronize immediately at $1 / \Lambda_{Q C D} \sim 10^{-24} \mathrm{~s}$. It is impossible to distinguish whether the light quark jet is from up-type or down-type quarks. The identical final states then lead to simultaneous measurements of the first two generation scalar quarks, and polarization effects of such chargino decays automatically average out. We conclude that the OSET can be directly applied to such studies.

However, the decay's final state of the stop is clearly identifiable [13]; we have

$\tilde{t}_{\mathrm{L}} \rightarrow b_{\mathrm{L}}\left(\tilde{\chi}_{1}^{+}\right)_{\mathrm{L}}, \quad \tilde{t}_{\mathrm{L}}^{*} \rightarrow(\bar{b})_{\mathrm{R}}\left(\tilde{\chi}_{1}^{-}\right)_{\mathrm{R}}$

\footnotetext{
The chargino mixing matrix $V_{i j}$ is as in [12].

${ }^{2}$ Since the scalar propagator does not carry any spin information, the two fermion lines connected by a scalar mediator have no correlation in spin. This effect does not depend on whether the scalar is on-shell or off-shell. The spin correlation is only at the scalar vertex as discussed. If squarks are heavier than the gluino, in gluino three-body decay $\tilde{g} \rightarrow$ $\bar{u} d \tilde{\chi}_{1}^{+}$, the polarization is identical to the case when squarks are on-shell. Hence, the above discussion of the chargino polarization in on-shell squarks states applies to the off-shell squark cases.
}
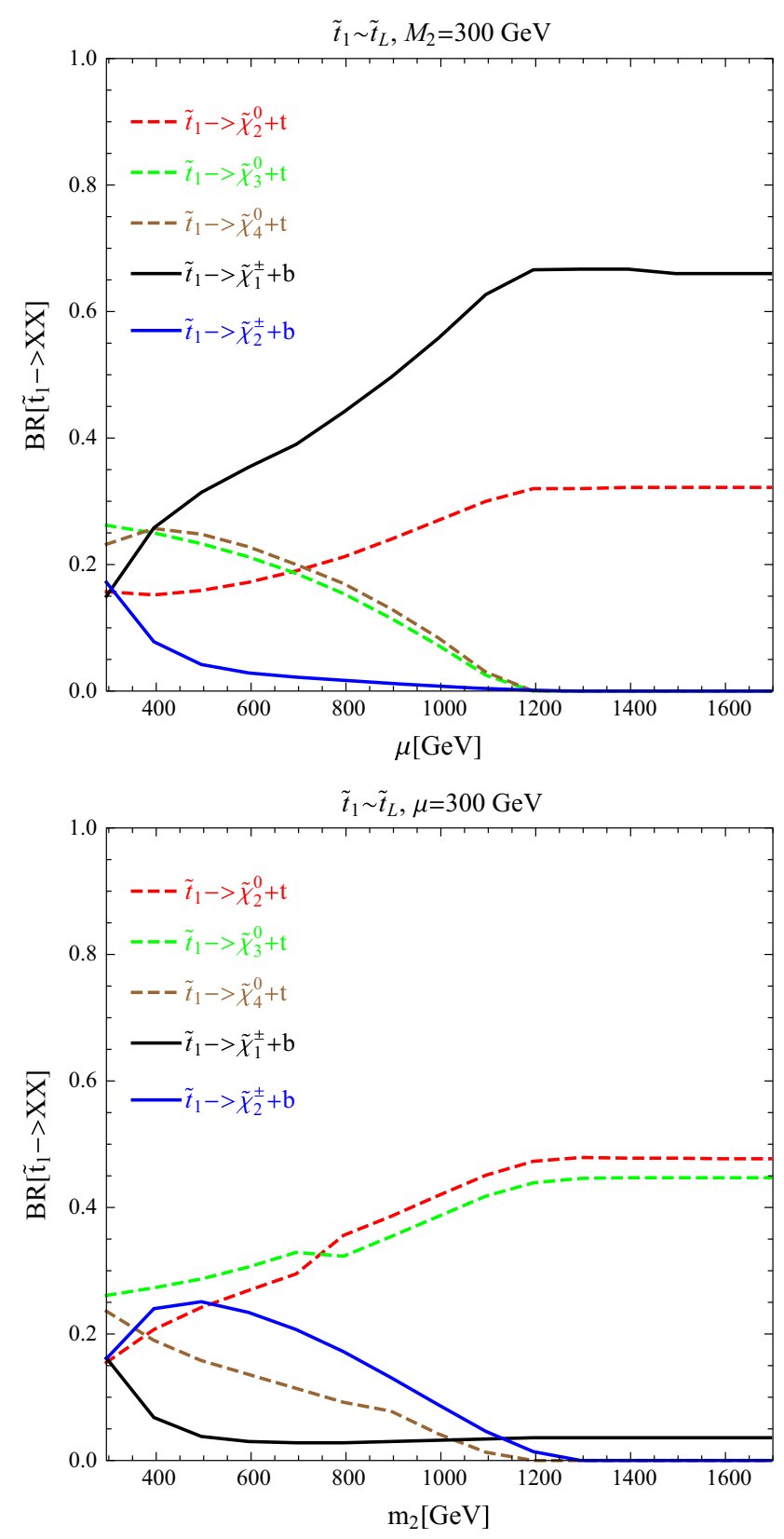

Fig. 3 Decay branching ratio of lightest stop $\tilde{t}_{1}$ for left stop with winolike or Higgsino-like chargino

The argument then applies to the stop search. A large leftright mixing $A$-term in the third generation often results in significant mixing in the stop states. We look into all three cases of the lightest stop $\tilde{t}_{1}$ being left, right or of large mixing in Figs. 3, 4, 5 with varied chargino states. The realization of the $125 \mathrm{GeV}$ Higgs in MSSM may put stringent constraints over the choices of $X_{t}=A_{t}-\mu \cot \beta$ and the stop mass. However, NMSSM with an additional singlet scalar provides more freedom and, therefore, we do not make the specific assumption of having $125 \mathrm{GeV}$ Higgs in this study but take a signal-driven approach. 

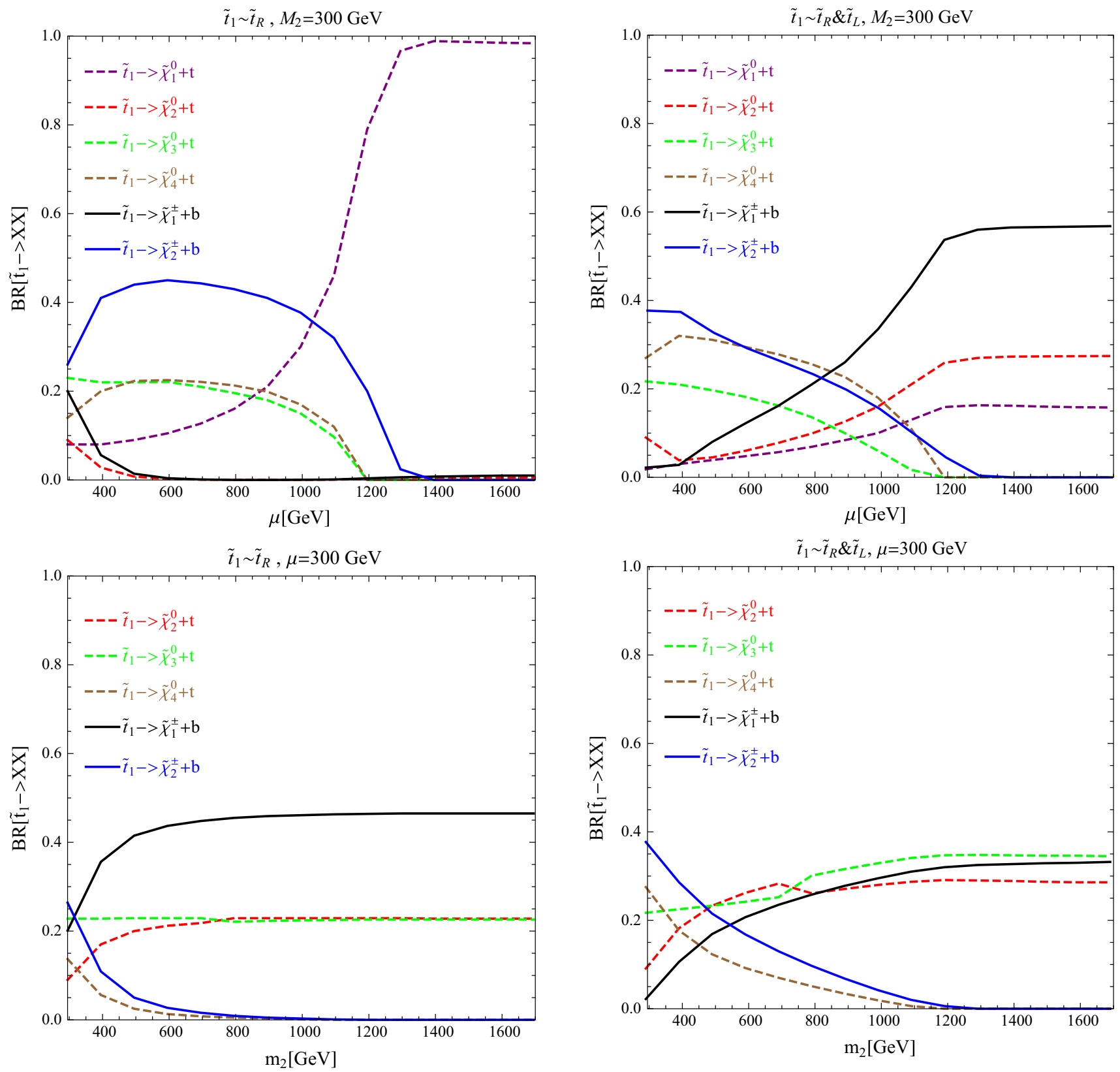

Fig. 4 Decay branching ratio of lightest stop $\tilde{t}_{1}$ for right stop with wino-like or Higgsino-like chargino

In Figs. 3 and 5, when $\tilde{t}_{1}$ has a significant portion of left stop state, the wino-like chargino dominates the stop decay as $\tilde{t}_{1} \rightarrow b \tilde{\chi}^{+}$.

As shown in Fig. 4, the right stop is dominated by the $\tilde{t}_{1} \rightarrow t \tilde{\chi}_{1}^{0}$ decay. Such final states with on-shell top quarks have been widely studied [13-15]. In the right polarized top quark case, the leptons are moving in the direction of the top boost direction as in Fig. 6, and we therefore expect that the lepton $p_{\mathrm{T}}$ is harder when taking into account the polarization information. The lepton cut efficiencies show a significant enhancement. In Table 1, a benchmark point of $1.3 \mathrm{TeV}$ for

Fig. 5 Decay branching ratio of lightest stop $\tilde{t}_{1}$ for left-right mixed stop with wino-like or Higgsino-like chargino

the right $\tilde{t}_{1}$ also confirms the argument. Comparing with the kinematics-only studies, the enhancement with polarization effects can be as large as $17 \%$.

\section{Polarized chargino}

In this session, we focus on the polarized chargino decay. The direct search of the chargino via tri-lepton channels at LHC [16] constrained the chargino mass to be over $350 \mathrm{GeV}$ for the massless $\tilde{\chi}_{1}^{0}$ limit. On the other hand, the bound is certainly weaker when the mass difference between chargino 
and lightest neutralino is smaller. We use two un-excluded benchmark points to illustrate the feature. One benchmark is the light chargino with a nearly degenerate spectrum where $M_{\tilde{\chi}_{1}^{+}}-M_{\tilde{\chi}_{1}^{0}} \simeq 50 \mathrm{GeV}$ with $M_{\tilde{\chi}_{1}^{+}} \simeq 250 \mathrm{GeV}$. The second benchmark is $M_{\tilde{\chi}_{1}^{+}} \simeq 285 \mathrm{GeV}$ and the same bino mass as the first one so that $M_{\tilde{\chi}_{1}^{+}}-M_{\tilde{\chi}_{1}^{0}}>m_{W}$ and $W$ is on-shell. We take three benchmark scenarios to illustrate the feature with $\tilde{\chi}_{1}^{ \pm}$ being pure wino, pure Higgsino, and mixed wino-Higgsino.

\subsection{Wino-like chargino}

In the case of the wino-like chargino, we use the above two benchmarks (off-shell $W$ and on-shell $W$ ) to illustrate the feature. For the first benchmark point of chargino three-body decay,

$\tilde{\chi}_{1}^{+} \rightarrow \tilde{\chi}_{1}^{0}+e^{+}+v_{e}$

it can be realized by the three processes shown in Fig. 7 with slepton mediation and Fig. 8 of the $W$ mediation.

In principle, interference among all three processes is important and, in fact, the three diagrams do destructively interfere. However, to qualitatively see the feature, we discuss the extreme cases where one single diagram completely dominates the three-body decay by which one can understand the qualitative feature at least in some decoupling limit. In

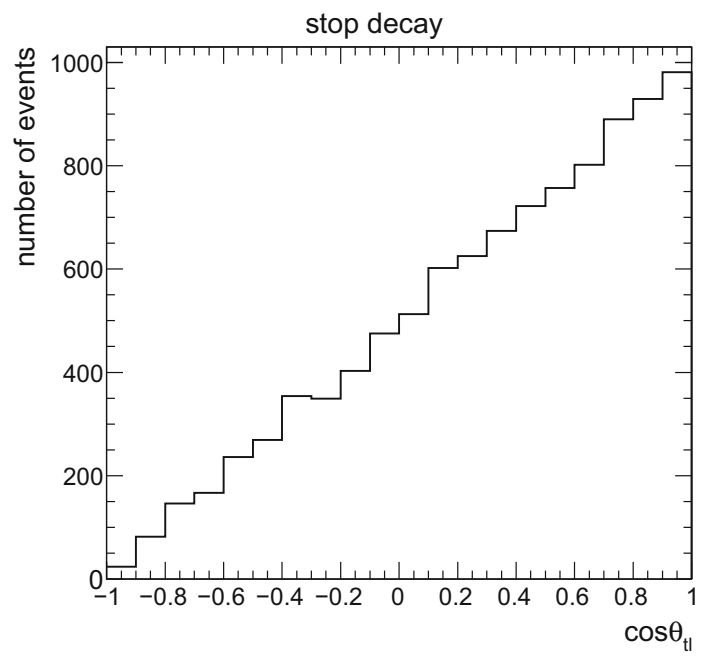

Fig. 6 Normalized distribution $1 / \Gamma d \Gamma / d \cos \theta$ where $\theta$ is the angle between the charged lepton and the right-handed top-quark movement direction

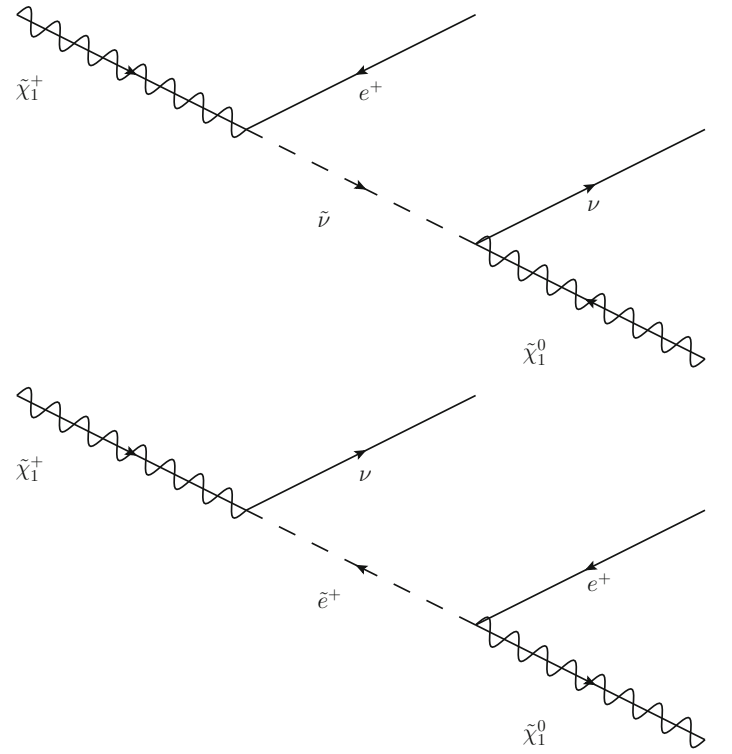

Fig. $7 \quad \tilde{\chi}_{1}^{+} \rightarrow e^{+} v_{e} \tilde{\chi}_{1}^{0}$ via sleptons

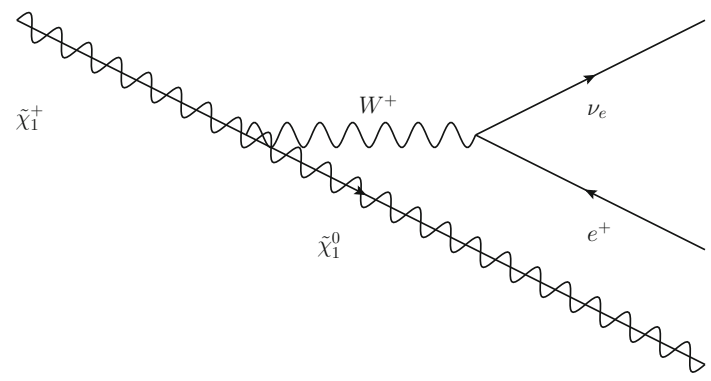

Fig. $8 \tilde{\chi}_{1}^{+} \rightarrow e^{+} v_{e} \tilde{\chi}_{1}^{0}$ via $W^{+}$

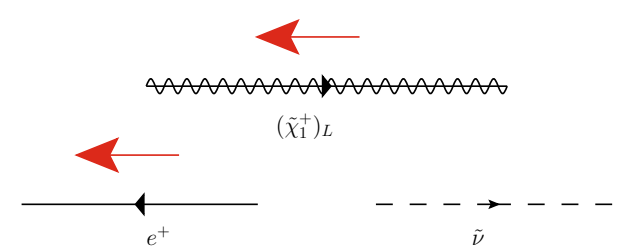

Fig. 9 Spin correlation in left-handed $\tilde{\chi}_{1}^{+}$decay into sneutrino $\tilde{v}$

sneutrino mediation as in Fig. 7a, sneutrino propagator does not carry any spin, so the spin direction of the positron is identical to the initial chargino spin. Only a right-handed positron participates in the weak interaction and, therefore, the positron moves in the opposite direction of the initial chargino as shown in Fig. 9.
Table 1 Lepton $p_{\mathrm{T}}$ cut efficiencies for $1.3 \mathrm{TeV}$ $\tilde{t}_{1} \rightarrow t \tilde{\chi}_{1}^{0}$, results with polarization effects or kinematics only

\begin{tabular}{lllll}
\hline$M_{\tilde{t}_{1}}$ & Category & $\begin{array}{l}p_{\mathrm{T}}>20 \mathrm{GeV} \\
(\%)\end{array}$ & $\begin{array}{l}p_{\mathrm{T}}>25 \mathrm{GeV} \\
(\%)\end{array}$ & $\begin{array}{l}p_{\mathrm{T}}>30 \mathrm{GeV} \\
(\%)\end{array}$ \\
\hline $1.3 \mathrm{TeV}$ & Polarized & 96.9 & 95.3 & 93.5 \\
& Kinematic & 89.0 & 85.4 & 81.8 \\
\hline
\end{tabular}




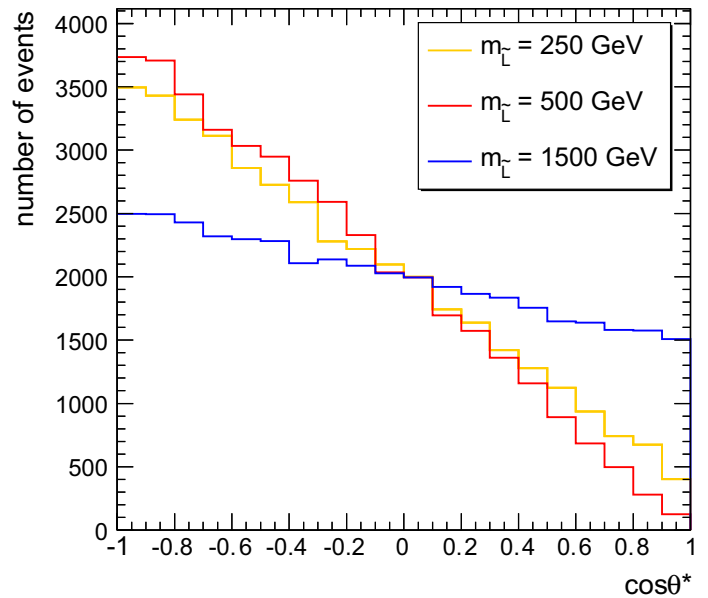

Fig. 10 Normalized distribution $1 / \Gamma d \Gamma / d \cos \theta$ where $\theta$ is the angle between charged lepton and chargino direction of the movement in the reconstructed $\chi_{1}^{+}$frame for $\Delta M=50 \mathrm{GeV}$ with different slepton masses $M_{\tilde{\ell}}$

In selectron mediation as in Fig. $7 \mathrm{~b}$, the electron decay from the spin-0 selectron does not correlate with the initial polarization of the chargino in both the on-shell and the offshell selectron cases. The electron is therefore completely universally distributed in space.

For $W$-mediation, the situation then depends on whether $W$ is on-shell or off-shell. For the first benchmark point, $W$ is off-shell. It is well known that there exist four polarizations in the off-shell $W$ case, including an additional scalar contribution as well as the scalar-longitudinal interference contribution [17]. On the other hand, the pseudoscalar contribution is proportional to the final state mass square, which is similar to charged pion decay. Then the charm and $\tau$ dominate the scalar contribution of the offshell $W$. Leptons are then much softer from $\tau$ three-body decay.

The contributions also destructively interfere with each other. Figure 10 shows the angle $\theta$ between the positron and chargino in the reconstructed chargino rest frame for three choices of the slepton masses. For simplicity, we assume $M_{\tilde{\ell}}=M_{\tilde{e}}$ with negligible lepton $A$-terms. $M_{2}$ is fixed at $250 \mathrm{GeV}$, while $M_{1}$ is taken to be $200 \mathrm{GeV}$. For $M_{\tilde{\ell}}=250$ and $500 \mathrm{GeV}$, the sneutrino and selectron contributions are both significant. Since the bino is a SM singlet, the $W$ contribution is only realized through wino-bino mixing, which is small in our benchmark points. Therefore, we find that the slepton contribution does not decouple even when $M_{\tilde{\ell}}=1.5 \mathrm{TeV}$.

When $W$ is on-shell for the second benchmark point, the two-body decay contribution completely takes over and we plot $\cos \theta$ for the on-shell $W$ case in Fig. 11 .

The positron is always moving in the opposite direction to the left-handed polarized chargino.

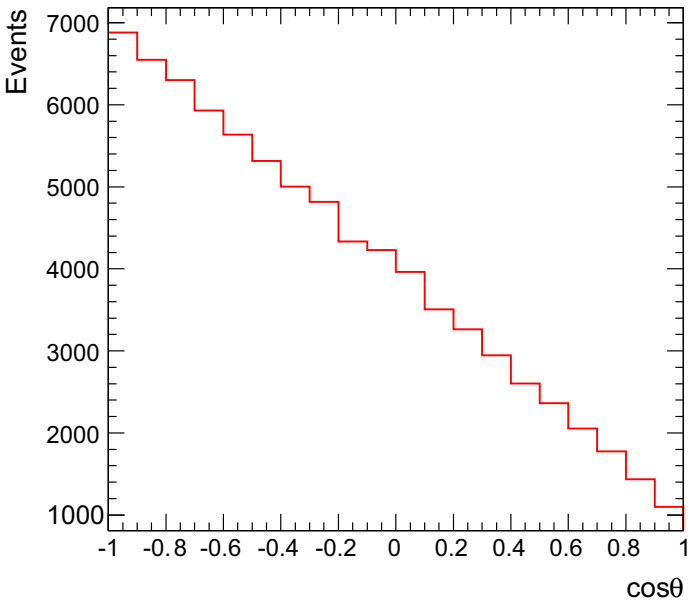

Fig. 11 Normalized distribution $1 / \Gamma d \Gamma / d \cos \theta$ where $\theta$ is the angle between the charged lepton and the chargino direction of the movement in a reconstructed $\chi_{1}^{+}$frame for the on-shell $W$ case

\subsection{Higgsino-like chargino}

The Higgsino couples to the squarks via Yukawa couplings and hence the squarks of the first two generations mostly decay into wino states. The third generation squarks, stop or sbottom, of relatively large $\tan \beta$, can dominantly decay into Higgsino states. Equation (7) gives the interaction between the stop and charginos,

$$
\begin{aligned}
\mathcal{L}_{b \tilde{t} \tilde{\chi}^{ \pm}}= & {\left[-g V_{i 1} \tilde{t}_{\mathrm{L}}+y_{t} V_{i 2} \tilde{t}_{\mathrm{R}}\right]\left(\bar{b} P_{\mathrm{R}} \tilde{\chi}_{i}^{+c}\right) } \\
& +y_{b} U_{i 2}^{*} \tilde{t}_{\mathrm{L}}\left(\bar{b} P_{\mathrm{L}} \tilde{\chi}_{i}^{+c}\right),
\end{aligned}
$$

where $U_{i j}$ and $V_{i j}$ are the standard chargino mixing matrices as in [12]. If $\tilde{\chi}_{1}^{ \pm}$is dominated by the Higgsino component and $y_{t}$ is much larger than $y_{b}$, the $\tilde{\chi}_{1}^{ \pm}$polarization in the decay is identical to the case of the wino, $\tilde{\chi}_{1}^{ \pm}$. Only for $\tan \beta \sim$ 30 or greater, the bottom Yukawa $y_{b}$ is close to $y_{t}$, and the $\tilde{\chi}_{1}^{ \pm}$polarization then has both significantly left-handed and right-handed portions. However, a supersymmetric scenario with large $\tan \beta$ is severely constrained by rare decay process like $B_{\mathrm{S}} \rightarrow \mu^{+} \mu^{-}$. For the small $\tan \beta$ cases, the Higgsino behavior is then similar to the wino. On the other hand, since the electron/muon Yukawa couplings are negligible Higgsino decay into lepton is only an effect of the on-shell or off-shell $W$, which has been discussed in the last part of the wino case.

\subsection{Mixed Wino-Higgsino}

In the case of mixed wino-Higgsino where $M_{2} \sim \mu$, since the Higgsino decay partial width is much larger than the wino case, the mixed wino-Higgsino is also Higgsino-like. Figure 12 plots the normalized angular distribution for pure wino with $500 \mathrm{GeV}$ sleptons, pure Higgsino, and mixed wino- 


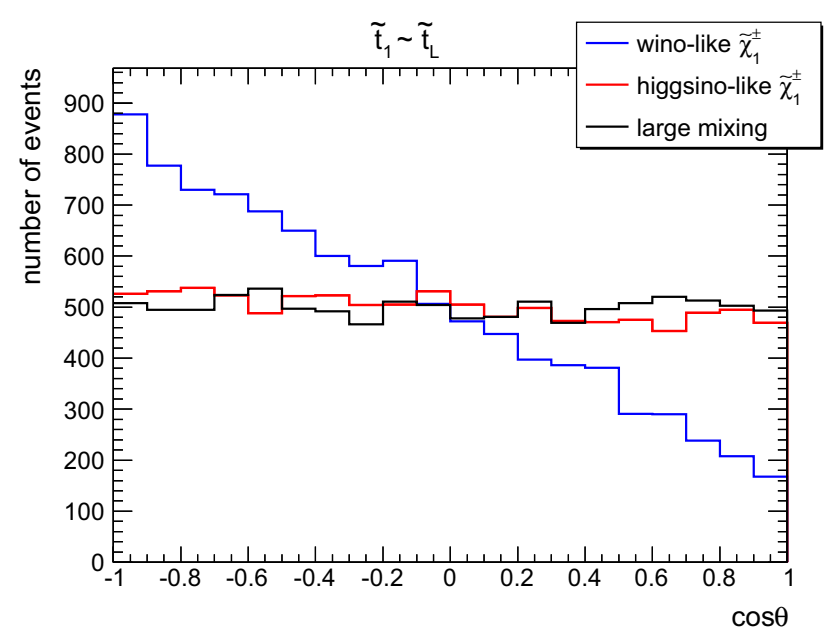

Fig. 12 Normalized distribution $1 / \Gamma d \Gamma / d \cos \theta$ where $\theta$ is the angle between charged lepton and chargino direction of the movement in a reconstructed $\chi_{1}^{+}$frame for the chargino three-body decay with

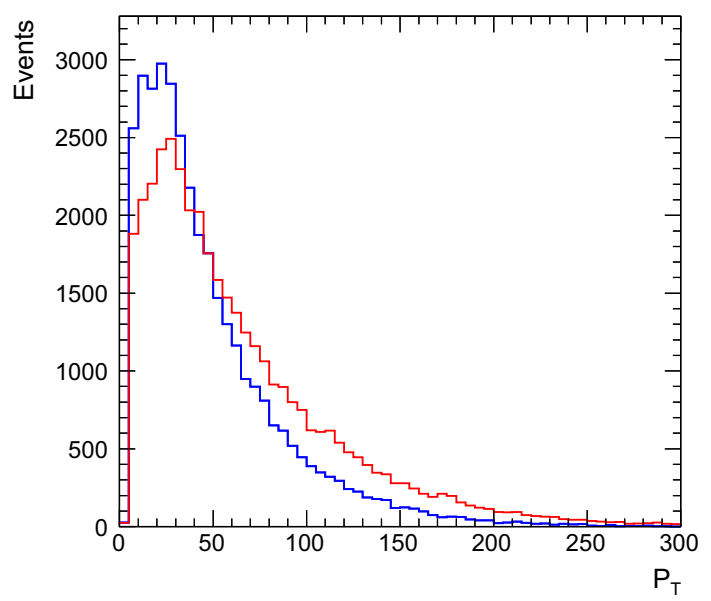

Fig. 13 Lepton $p_{\mathrm{T}}$ distribution of chargino decay $\tilde{\chi}_{1}^{ \pm} \rightarrow \ell^{ \pm} \nu \tilde{\chi}_{1}^{0}$ in a comparison of the full matrix element method with the kinematic method. A blue line is the full matrix element result from MadEvent, while a red line is the kinematic decay from Pythia

Higgsino. It is clear that the mixed wino-Higgsino situation is similar to a Higgsino-like chargino.

\section{Lepton in cascade decay chain}

As discussed in the previous section, a wino-like chargino with a light slepton or on-shell $W$ decaying into lepton final states may show a significant anti-boost effect. Consequently, it may make the leptons softer in the lab frame than the ones in the chargino rest frame. Since it is a boost effect, the effect is visible only when the chargino has a significant kinematic boost. If the chargino is produced nearly at rest, which corresponds to the scenario with a small mass difference between the chargino and stop/gluino, the effect is then tiny. On the other hand, if the mass difference between the chargino and
Table 2 Chargino benchmark points of the wino with light sleptons, the wino with decoupled sleptons, and the Higgsino with intermediate $\tan \beta$. For the case of a wino with on-shell $W$, the situation is similar to a wino with a light slepton as discussed

\begin{tabular}{llll}
\hline Scenarios & $\begin{array}{l}M_{\tilde{\chi}_{1}^{ \pm}} \\
(\mathrm{GeV})\end{array}$ & $\begin{array}{l}M_{\tilde{\chi}_{1}^{0}} \\
(\mathrm{GeV})\end{array}$ & $\begin{array}{l}M_{\tilde{\ell}} \\
(\mathrm{GeV})\end{array}$ \\
\hline A-wino with light sleptons & 250 & 200 & 500 \\
B-wino decoupled sleptons & 250 & 200 & 2000 \\
C-Higgsino $(\tan \beta=25)$ & 250 & 200 & N/A \\
\hline
\end{tabular}

stop/gluino is too large, the chargino is highly boosted in the system and the lepton momentum in the lower end is then completely dominated by the boost effect. Since we are only looking at the consequence of a lepton $p_{\mathrm{T}}$ cut which only affects the softer leptons, the effect is only significant in some intermediate mass range.

Figure 13 shows the lepton $p_{\mathrm{T}}$ distribution of chargino decay $\tilde{\chi}_{1}^{ \pm} \rightarrow \ell^{ \pm} v \tilde{\chi}_{1}^{0}$ in a comparison of the full matrix element method with the kinematic method for $M_{\tilde{t}}=1.3 \mathrm{TeV}$ and $M_{\tilde{\chi}_{1}^{ \pm}}=250 \mathrm{GeV}$. A blue line is the full matrix element result from MadEvent [18], while a red line is the kinematic decay from Pythia [19], which shows that the full matrix element result is significantly softer than the result from kinematic decay.

We take three chargino benchmark points to illustrate the feature, respectively, as shown in Table 2.

We listed the cut survival probabilities with different $p_{\mathrm{T}}$ cuts, respectively, in Table 3 . The benchmark points are listed as (A) wino with light sleptons; (B) wino with decoupled sleptons, and (C) Higgsino (with intermediate $\tan \beta=25$ ) shown in Table 2 and the top squark mass is taken to be $700 \mathrm{GeV}$, which is around the current bound from LHC.

In Table 4, we take the chargino scenario of a wino with a light slepton, which shows the most significant boost effects, as shown in Table 3, studying the effects with different top squark masses and collision energies.

The most significant reductions of the efficiencies are about $25 \%$. Therefore, if one only uses an on-shell effective field theory approach for this search, the signal is actually underestimated.

\section{Conclusions}

We study the polarization effects in supersymmetry searches of multi-jets plus leptons final states. We find it justifiable for the first two generations to only use on-shell effective theories. While for measurements related to third generation squarks, for instance, the polarization effect of the charginos from a stop may reduce the lepton $p_{\mathrm{T}}$ cut efficiencies in cross section measurements by $25 \%$ when the slepton contributions dominate in the chargino decay or the $W$ are on-shell. 
Table 3 Lepton $p_{\mathrm{T}}$ cut survival probability for three chargino scenarios when top squark is taken to be $700 \mathrm{GeV}$. The category named "polarized" is for the situation with polarization taken into account, while "kinematic" stands for the kinematic decay cases

Table 4 Lepton $p_{\mathrm{T}}$ cut survival probability for a wino with light sleptons for different top squark benchmark points and collision energies. The category named "polarized" is for a situation with polarization taken into account, while "Kinematic" stands for the kinematics-only decay cases

\begin{tabular}{|c|c|c|c|c|c|}
\hline$M_{\tilde{t}}$ & $\tilde{\chi}_{1}^{ \pm}$ & Category & $\begin{array}{l}p_{\mathrm{T}}>20 \mathrm{GeV} \\
(\%)\end{array}$ & $\begin{array}{l}p_{\mathrm{T}}>25 \mathrm{GeV} \\
(\%)\end{array}$ & $\begin{array}{l}p_{\mathrm{T}}>30 \mathrm{GeV} \\
(\%)\end{array}$ \\
\hline \multirow[t]{6}{*}{$700 \mathrm{GeV}$} & \multirow[t]{2}{*}{$\tilde{W}(\mathrm{~A})$} & Polarized & 42.4 & 34.7 & 28.1 \\
\hline & & Kinematic & 52.9 & 45.9 & 39.4 \\
\hline & \multirow[t]{2}{*}{$\tilde{W}(\mathrm{~B})$} & Polarized & 51.7 & 44.9 & 38.3 \\
\hline & & Kinematic & 55.7 & 48.1 & 41.1 \\
\hline & \multirow[t]{2}{*}{$\tilde{H}(\mathrm{C})$} & Polarized & 53.6 & 46.3 & 39.6 \\
\hline & & Kinematic & 55.0 & 47.7 & 40.9 \\
\hline
\end{tabular}

\begin{tabular}{|c|c|c|c|c|c|}
\hline$\sqrt{s} @ \mathrm{LHC}$ & $M_{\tilde{t}}$ & Category & $\begin{array}{l}p_{\mathrm{T}}>20 \mathrm{GeV} \\
(\%)\end{array}$ & $\begin{array}{l}p_{\mathrm{T}}>25 \mathrm{GeV} \\
(\%)\end{array}$ & $\begin{array}{l}p_{\mathrm{T}}>30 \mathrm{GeV} \\
(\%)\end{array}$ \\
\hline \multirow[t]{2}{*}{$8 \mathrm{TeV}$} & \multirow[t]{2}{*}{$700 \mathrm{GeV}$} & Polarized & 42.4 & 34.7 & 28.1 \\
\hline & & Kinematic & 52.9 & 45.9 & 39.4 \\
\hline \multirow[t]{2}{*}{$8 \mathrm{TeV}$} & \multirow[t]{2}{*}{$1 \mathrm{TeV}$} & Polarized & 48.2 & 41.6 & 35.6 \\
\hline & & Kinematic & 59.5 & 53.8 & 48.3 \\
\hline \multirow[t]{2}{*}{$10 \mathrm{TeV}$} & \multirow[t]{2}{*}{$1.3 \mathrm{TeV}$} & Polarized & 53.2 & 47.3 & 41.8 \\
\hline & & Kinematic & 63.5 & 58.6 & 54.1 \\
\hline \multirow[t]{2}{*}{$10 \mathrm{TeV}$} & \multirow[t]{2}{*}{$1.5 \mathrm{TeV}$} & Polarized & 55.3 & 49.7 & 44.4 \\
\hline & & Kinematic & 65.4 & 61.1 & 56.7 \\
\hline \multirow[t]{2}{*}{$14 \mathrm{TeV}$} & \multirow[t]{2}{*}{$1.3 \mathrm{TeV}$} & Polarized & 53.6 & 47.9 & 42.3 \\
\hline & & Kinematic & 63.7 & 59.0 & 54.1 \\
\hline \multirow[t]{2}{*}{$14 \mathrm{TeV}$} & \multirow[t]{2}{*}{$1.5 \mathrm{TeV}$} & Polarized & 56.2 & 50.9 & 45.6 \\
\hline & & Kinematic & 66.0 & 61.5 & 57.1 \\
\hline
\end{tabular}

The signal is then underestimated if only an on-shell effective theory approach is taken in the simulation of the signal, and the real bound on the squark/gluino should be more stringent. In the other limit, when we have the right stop $\tilde{t}_{1} \rightarrow t \tilde{\chi}_{1}^{0}$, the polarization effect of a lepton from a right polarized top quark may enhance the lepton $p_{\mathrm{T}}$ cut efficiencies in a cross section measurement by maximally $17 \%$.

Acknowledgments The project is supported in part by the National Science Foundation of China $(11275168,11422544)$ and by the Zhejiang University Fundamental Research Funds for the Central Universities as well as Zhejiang University K.P.Chaos High Technology Development Foundation.

Open Access This article is distributed under the terms of the Creative Commons Attribution 4.0 International License (http://creativecomm ons.org/licenses/by/4.0/), which permits unrestricted use, distribution, and reproduction in any medium, provided you give appropriate credit to the original author(s) and the source, provide a link to the Creative Commons license, and indicate if changes were made. Funded by SCOAP ${ }^{3}$.

\section{References}

1. N. Arkani-Hamed, P. Schuster, N. Toro, J. Thaler, L.-T. Wang, B. Knuteson, S. Mrenna, arXiv:hep-ph/0703088

2. L.D. Landau, Dokl. Akad. Nauk Ser. Fiz. 60, 207 (1948)

3. C.-N. Yang, Phys. Rev. 77, 242 (1950)
4. G. Aad et al. [ATLAS Collaboration], son with the ATLAS detector at the LHC, Phys. Lett. B arXiv:1207.7214 [hep-ex]

5. S. Chatrchyan et al. [CMS Collaboration], the LHC, Phys. Lett. B arXiv:1207.7235 [hep-ex]

6. The ATLAS collaboration, ATLAS-CONF-2013-062

7. J.F. Hirschauer [CMS Collaboration], PoS ICHEP 2012, 177 (2013)

8. B.K. Bullock, K. Hagiwara, A.D. Martin, Phys. Rev. Lett. 67, 3055 (1991)

9. G.L. Kane, G.A. Ladinsky, C.P. Yuan, Phys. Rev. D 45, 124 (1992)

10. X.Q. Li, Z.G. Si, K. Wang, L. Wang, L. Zhang, G. Zhu, Phys. Rev. D 89, 077703 (2014). arXiv:1311.6874 [hep-ph]

11. A. Czarnecki, J.G. Korner, J.H. Piclum, Phys. Rev. D 81, 111503 (2010). arXiv:1005.2625 [hep-ph]

12. J.F. Gunion, H.E. Haber, Nucl. Phys. B 278, 449 (1986)

13. I. Low, Phys. Rev. D 88, 095018 (2013). arXiv:1304.0491 [hep-ph]

14. J. Shelton, Phys. Rev. D 79, 014032 (2009). arXiv:0811.0569 [hep$\mathrm{ph}]$

15. M. Perelstein, A. Weiler, JHEP 0903, 141 (2009). arXiv:0811.1024 [hep-ph]

16. [ATLAS Collaboration], ATLAS-CONF-2013-035

17. S. Groote, J.G. Korner, P. Tuvike, Eur. Phys. J. C 73, 2454 (2013). arXiv:1301.0881 [hep-ph]

18. J. Alwall, M. Herquet, F. Maltoni, O. Mattelaer, T. Stelzer, JHEP 1106, 128 (2011). arXiv:1106.0522 [hep-ph]

19. T. Sjostrand, S. Mrenna, P.Z. Skands, JHEP 0605, 026 (2006). arXiv:hep-ph/0603175 